\title{
Label-Free in Situ Optical Monitoring of the Adsorption of Oppositely Charged Metal Nanoparticles
}

\author{
Beatrix Peter, ${ }^{\dagger \dagger}$ Sandor Kurunczi, ${ }^{\ddagger}$ Daniel Patko, ${ }^{\dagger, \ddagger}$ Istvan Lagzi, ${ }^{\S}$ Bartlomiej Kowalczyk, ${ }^{\|}$Zoltán Rácz, ${ }^{\perp}$ \\ Bartosz A. Grzybowski, ${ }^{*}, \|$ and Robert Horvath*, \\ ${ }^{\dagger}$ Doctoral School of Molecular- and Nanotechnology, University of Pannonia, H-8201 Veszprém, Hungary \\ ${ }^{\ddagger}$ Nanobiosensorics Group, Research Centre for Natural Sciences, Institute for Technical Physics and Materials Science, H-1121 \\ Budapest, Hungary \\ ${ }^{\S}$ Department of Physics, Budapest University of Technology and Economics, H-1111 Budapest, Hungary \\ "Department of Chemistry and Chemical and Biological Engineering, Northwestern University, Evanston, Illinois 60208, United \\ States \\ ${ }^{\perp}$ Research Group for Theoretical Physics of the Hungarian Academy of Sciences, Eötvös Loránd University, H-1053 Budapest, \\ Hungary
}

ABSTRACT: The mechanism of alternating deposition of oppositely charged gold nanoparticles (AuNPs) was investigated by optical waveguide lightmode spectroscopy (OWLS). OWLS allows monitoring of the kinetics of layer-by-layer (LbL) adsorption of positively and negatively charged nanoparticles in real time without using any labels so that the dynamics of layer formation can be revealed. Positively charged NPs that are already deposited on a negatively charged glass substrate strongly facilitate the adsorption of the negatively charged particles. The morphology of the adsorbed layer was also investigated with atomic force microscopy (AFM). AFM revealed that the interaction between oppositely charged particles results in the formation of NP clusters with sizes varying between 100 and 6000 NPs. The cluster size distribution is found to be an exponentially decaying function, and we propose a simple theory to explain this finding.

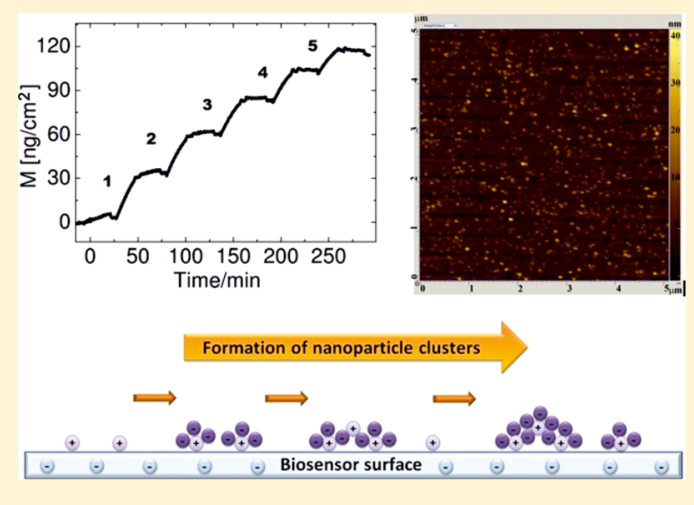

\section{INTRODUCTION}

Thin films comprising various types of nanoparticles (NPs) [e.g., fluorescent $(\mathrm{CdS}, \mathrm{CdSe})$, metallic $(\mathrm{Au}, \mathrm{Ag}, \mathrm{Cu})$, or oxide $\left(\mathrm{Fe}_{2} \mathrm{O}_{3}, \mathrm{TiO}_{2}\right)$ particles] have attracted considerable scientific attention due to their potential applications in corrosion protection, heterogeneous catalysis, optics, biomedicine, and biological sensors. ${ }^{1-7}$ There are several techniques [e.g., layerby-layer (LbL), electrochemical deposition, chemical or physical vapor deposition, and spray-based methods] that can be used to coat different substrates with nanoscopic components, thus creating thin films with tunable properties. $^{8-10} \mathrm{LbL}$ is a robust and cost-effective method to create films by deposition or adsorption via self-assembly of oppositely charged chemical species. ${ }^{11-17}$ Understanding the mechanism and the kinetics of film formation is necessary to engineer thin films with controllable thickness and chemical properties. Although the structure of the deposited NP layers is usually well characterized, much less is known about the dynamics of the adsorption processes. Here, we use optical waveguide lightmode spectroscopy (OWLS) to study adsorption kinetics of charged gold NPs onto glass substrates. We found that adsorbed positively charged particles facilitate the adsorption of negatively charged particles. This interaction leads to the formation of nanoparticle clusters, revealed by atomic force microscopy (AFM) analysis of the sensor surfaces. The cluster size distribution was measured to be an exponential function, and we found that this behavior can be explained by a simple theoretical model.

\section{MATERIALS AND METHODS}

2.1. Nanoparticles and Their Solutions. We used oppositely charged gold nanoparticles (average metal core diameter $5.5 \mathrm{~nm}$ and standard deviation $\sigma=15 \%$ ) stabilized with self-assembled monolayers of $N, N, N$-trimethyl-(11-mercaptoundecyl)ammonium chloride (TMA, positively charged) and mercaptoundecanoic acid (MUA; negatively charged and fully deprotonated at $\mathrm{pH} \sim 11$ ). The $\zeta$ potentials of positively and negatively charged nanoparticles are $45 \mathrm{mV}$ and -45 $\mathrm{mV}$, respectively. ${ }^{18,19}$ In a typical experiment, dilute oppositely charged NP solutions $(\mathrm{pH}=10.0)$ were allowed to flow through the OWLS cuvette positioned above the sensing area of the OWLS chip in an alternating way (starting with the positively charged NP solution). The OWLS chip was cleaned before the experiments with chromosulfuric acid. The concentrations used in experiments were $2.5 \times 10^{-6} \mathrm{mM}$ (refractive index 1.33141) for AuMUA NPs and $1.58 \times 10^{-6} \mathrm{mM}$ (refractive index 1.331 46) for AuTMA NPs, and the $\mathrm{pH}$ was adjusted to 10 prior to the experiments. The baseline was set and a consecutive

Received: July 25, 2014

Revised: October 15, 2014

Published: October 17, 2014 
washing process was performed with tetramethylammonium hydroxide solution (TMAOH, Sigma-Aldrich) $\mathrm{pH}=10$ (refractive index 1.331 43).

2.2. Integrated Optical Scanning. Optical waveguide lightmode spectroscopy (OWLS) is a label-free surface-sensitive technique that uses evanescent optical waves. ${ }^{20-26}$ During the experiment, linearly polarized light is coupled into a planar optical waveguide sensor chip (type OW2400, Microvacuum Ltd., Hungary) through a coupling grating. The OWLS instrument (BIOS210, Microvacuum Ltd.) records the effective refractive indices of the zeroth-order TE (transverse electric mode) and TM (transverse magnetic mode) polarized waveguide modes with a time resolution of $\sim 13 \mathrm{~s}$. Upon adsorption onto the sensor's surface, the effective refractive indices shift to higher values, allowing monitoring of the in situ kinetics of adsorption processes. OWLS has been mostly used to characterize surface adsorption properties in protein-substrate or protein-NP film interactions. $^{27,28}$ In the present study, we use this sensitive technique to reveal the adsorption kinetics of charged NPs onto a charged deposition substrate.

2.3. Cleaning Procedures Prior to Experiments. The cleaning method after each experiment was as follows. First, the chip was immersed in chromosulfuric acid and potassium hydroxide to purify its surface. The plastic cuvette and the fluidic system were treated by oxidative plasma (SPI Supplies Plasma Prep II) that removes organic capping (protecting) agents from the surface of NPs that remained from the previous experiment. This procedure ensured that we could easily wash away from the substrate's surface, using distilled water, any remaining unprotected NPs and prepare the cuvette and the fluidic system for subsequent experiments.

2.4. Surface Characterization by Atomic Force Microscopy. Atomic force microscopy (AIST-NT, DigiScope1000) in tapping mode with $\mu$ masch tips ( $\mu$ masch, NSC15/AIBS, tip radius $<10 \mathrm{~nm}$, resonant frequency ca. $325 \mathrm{kHz}$ ) was used to visualize nanoparticles adsorbed on the sensor's surface. The scanned areas were $5 \times 5 \mu \mathrm{m}$ or $2 \times 2 \mu \mathrm{m}$. In order to quantitatively evaluate the recorded images, a free image analyzing software (Gwyddion 2.3) was used.

\section{RESULTS AND DISCUSSION}

3.1. Characterization of Applied Solutions: Absorbance and Refractive Index Increments. Deposition and characterization experiments were carried out at different dilutions. A serial dilution from stock solutions of AuMUA and AuTMA gold NPs was performed, and a concentration of $\sim 10^{-6} \mathrm{mM}$ for both AuMUA and AuTMA NPs was found to be optimal for the deposition experiments. The refractive index of the solutions was measured on a table-top Rudolf Research J157 automatic refractometer. The concentration of the solutions was determined from the absorbance at $520 \mathrm{~nm}$ (Agilent 8453 spectrophotometer). Using the measured refractive indices and concentrations of the dilution series, we determined the refractive index increments of the solutions. ${ }^{20,21}$ This information was necessary to estimate the mass of the adsorbed particles, which was determined according to de Feijter's formula ${ }^{27}$ as $\mathrm{M}=d_{\mathrm{A}}\left(n_{\mathrm{A}}-n_{\mathrm{c}}\right) /(\mathrm{d} n / \mathrm{d} c)$, where $d_{\mathrm{A}}, n_{\mathrm{A}}$, and $n_{\mathrm{c}}$ are thickness of adlayer, refractive index of adlayer, and refractive index of cover layer, respectively, and the averaged refractive index increment of AuMUA and AuTMA was $\mathrm{d} n / \mathrm{d} c$ $=0.3845 \mathrm{~cm}^{3} / \mathrm{g}$.

3.2. Alternating Deposition of Positively and Negatively Charged Particles. In the first experiment, NP adsorption onto the bare waveguide surface was investigated. After the baseline was fixed with TMAOH, the NP solution was allowed to flow and was subsequently flushed with TMAOH. As expected, the negatively charged AuMUA particles did not adsorb at all on the negatively charged surface (data not shown).
Afterward, we alternately deposited AuTMA and AuMUA on the bare waveguide surface, starting with the positively charged AuTMA nanoparticles. After the baseline was fixed with injected TMAOH ( 20 min) (1), AuTMA solution was injected for $20 \mathrm{~min}$ (2), followed by flushing with TMAOH for $5 \mathrm{~min}$ (3). Then the AuMUA solution was injected for $20 \mathrm{~min}$ (4) and was again flushed with TMAOH for $5 \mathrm{~min}$ (5). The entire cycle was repeated four more times. Every deposition cycle clearly increased the deposited amount (see Figure 1).

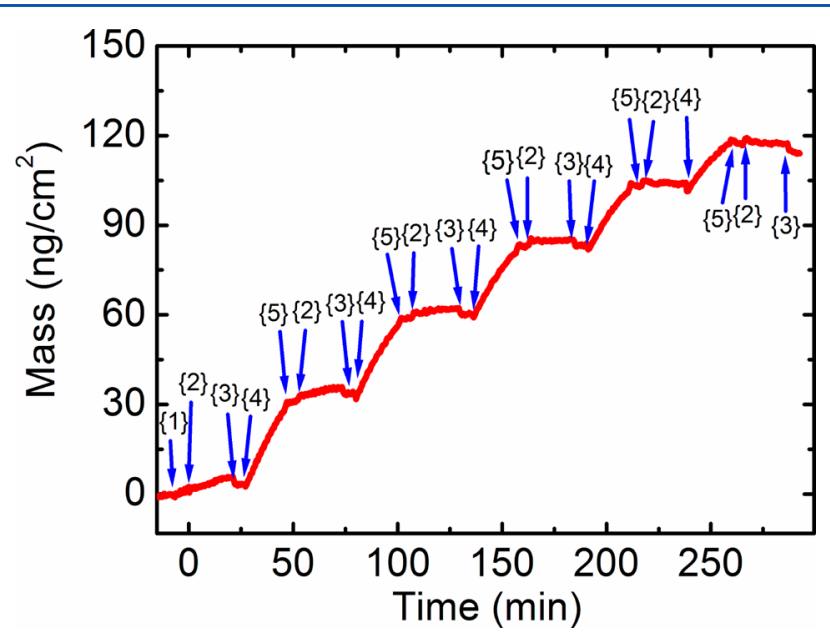

Figure 1. Surface adsorbed mass per unit area during alternating deposition of AuMUA and AuTMA particles: (1) Baseline with injected TMAOH, (2) AuTMA solution, (3) flushing with TMAOH, (4) AuMUA solution, and (5) flushing with TMAOH again.

As narrated above, at the beginning of each cycle, the positively charged AuTMAs were allowed to flow over the chip's negatively charged surface (2). Due to the electrostatic attractions, these NPs deposited onto the substrate, as reflected in the recorded curve in Figure 1. After any weakly adsorbed positively charged particles were washed away (3), negatively charged AuMUAs were introduced to the chip (4). The already-deposited AuTMA particles facilitated the adsorption of these negatively charged particles. A repeated flush (5) washed away any weakly adsorbed negatively charged particles. These consecutive cycles could be repeated several times, providing alternating deposition of oppositely charged NPs. Interestingly, with increasing deposition cycles, the initial slope of AuMUA adsorption in Figure 2 decreases, as the surface is covered with more particles and the number of binding sites available to

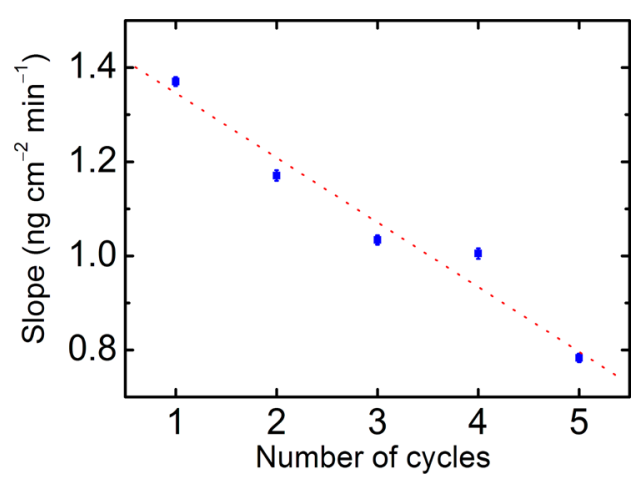

Figure 2. Dependence of relative initial slopes (rate constant for adsorption) of the deposition kinetic curve for AuMUA in each cycle. 
adsorb negatively charged particles decreases. This behavior contradicts the possible formation of distinct layers of nanoparticles deposited on each other (as in classical layerby-layer deposition observed for the alternating deposition of oppositely charged polyelectrolytes).

To investigate the morphology of the NP films, we examined the surface of the chip after deposition by AFM microscopy. Figure 3 shows AFM images of the waveguide sensor after the

\section{(a)}
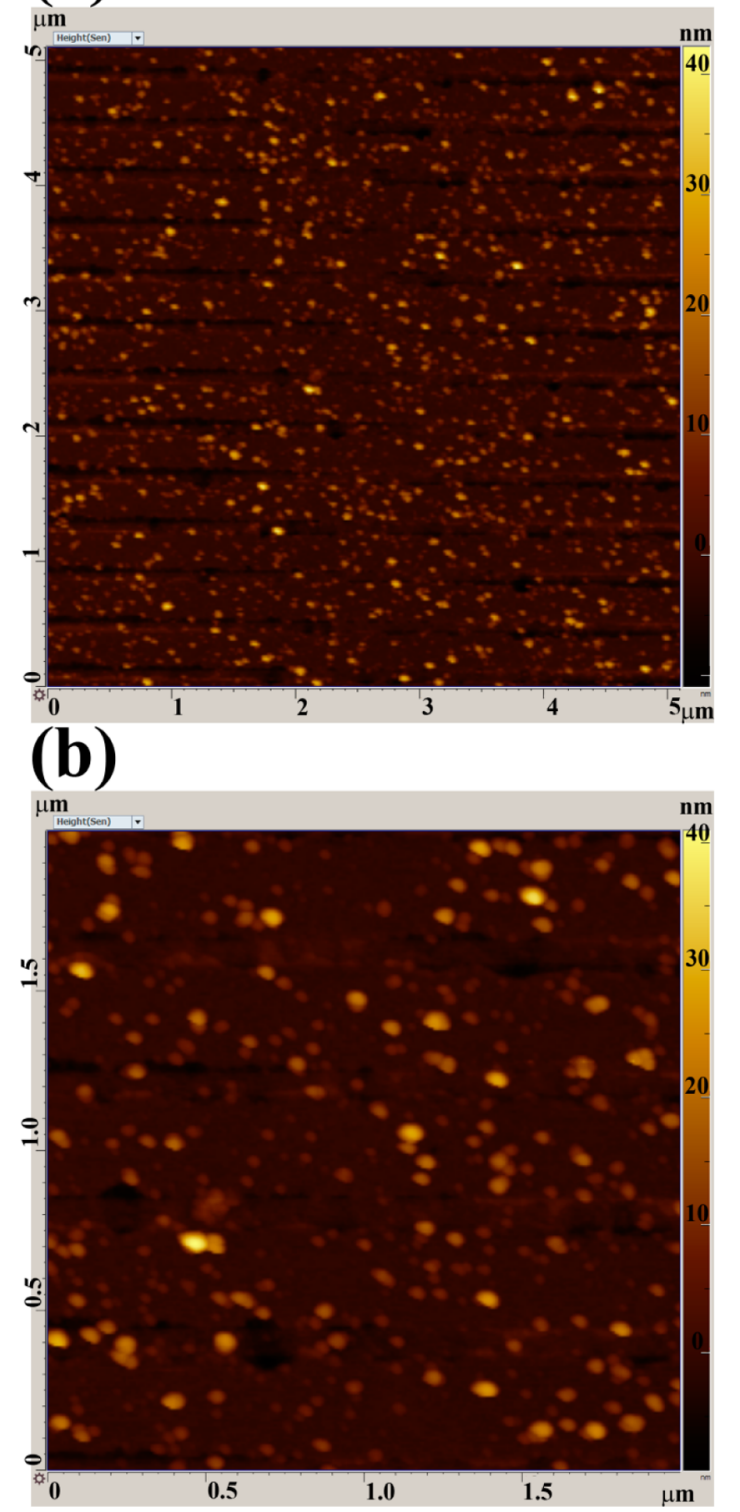

Figure 3. AFM image of the sensor surface after five deposition cycles of AuMUA and AuTMA particles: (a) $5 \mu \mathrm{m} \times 5 \mu \mathrm{m}$ imaged area; (b) magnified $2 \mu \mathrm{m} \times 2 \mu \mathrm{m}$ area.

adsorption experiment. The incoupling grating lines and the NP clusters are clearly seen. These AFM studies revealed that the deposited NPs form distinct patches/clusters rather than a continuous film. The density of NP clusters is 23.3 clusters/ $\mu \mathrm{m}^{2}$ and the number of clusters decreases approximately exponentially with cluster size (see Figure 4). The largest nanoparticle clusters have an area of around $23500 \mathrm{~nm}^{2}$, corresponding to a mean size of $\sim 153 \mathrm{~nm}$, whereas the smallest

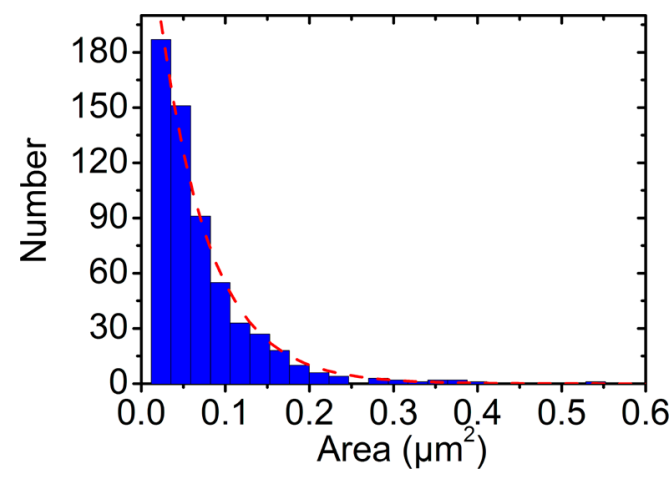

Figure 4. Number of NP clusters versus their area obtained from AFM measurements (Figure 3). The cluster size distribution follows an exponential function (red dashed curve).

clusters have an area of around $2350 \mathrm{~nm}^{2}$ with mean sizes of $\sim 48 \mathrm{~nm}$. The height of the clusters ranges from 5 to $35 \mathrm{~nm}$ depending on the area of the clusters. Comparing these dimensions with the average diameter of one nanoparticle $(\sim 5$ $\mathrm{nm})$, one can conclude that the largest clusters contain approximately 5000-6000 nanoparticles and even the smallest clusters contain some hundreds of nanoparticles.

Although the aggregation dynamics is presumably rather complex due to the long-range interactions between the charged NPs, the exponential cluster size distribution (frequently emerging in elementary stochastic processes) ${ }^{29}$ may have a simple explanation. Indeed, let us consider the cycle when the liquid contains positively charged NPs that attach either to the negatively charged surface or to the clusters negatively charged in the previous cycle. Since the aggregation is governed by the charges of the clusters, one can assume that the size of the clusters does not matter in the rate of attachment of a particle; that is, one may suppose that a particle attaches with equal probability to any of the existing clusters. Then the rate equation for the time evolution of the number of clusters $N_{k}$ containing $k$ NPs is given by

$$
\begin{gathered}
\dot{N}_{k}=-\frac{\alpha}{N} N_{k}+\frac{\alpha}{N} N_{k-1} \\
\dot{N}_{1}=-\frac{\alpha}{N} N_{1}+\beta
\end{gathered}
$$

where $N$ is the total number clusters $N=\sum_{k} N_{k}$, while $\alpha / N$ is the rate of attachment of a particle to a cluster (note that $1 / N$ appears in the rate since there is equal probability of attachment to any of the clusters). The parameter $\beta$ sets the rate of formation of a new cluster on the surface.

An easily understandable feature of eq 1 is that the number of clusters is determined by the rate of formation of new clusters, i.e. $N=\beta t$. One can also derive the solution of eq 1 by assuming a linear time dependence for the cluster numbers, $N_{k}(t)=n_{k} t$, leading to a recursion relation $n_{k}=\alpha n_{k-1} /(\alpha+\beta)$ with $n_{1}=\beta^{2} /(\alpha+\beta)$. The solution to this recursion is a cluster number distribution that decays exponentially with $k$ :

$$
N_{k}=n_{k} t=A \mathrm{e}^{-B k} t
$$

where $A=\beta^{2} /(\alpha+\beta)$ and $B=\ln (1+\beta / \alpha)$. Note that, in addition to the observed exponential decay (Figure 4 ), the above result is also in agreement with the general linear trend in $t$ observed in Figure 1 for the total adsorbed mass $M=m_{0} \sum_{k}$ $k n_{k} t$, where $m_{0}$ is the mass of an NP. It is clear that in order to account for the changes in the rate of mass adsorption in 


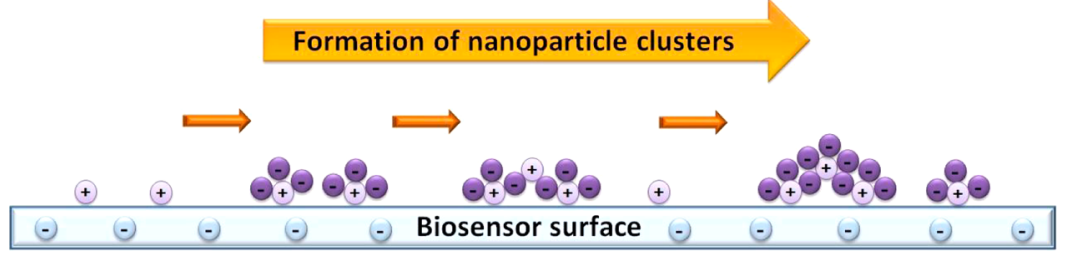

Figure 5. Illustration of nanoparticle cluster buildup from oppositely charged particles.

alternating deposition cycles, one would need a more sophisticated model that reflects the difference in the dynamics of positive and negative charges due to the negatively charged surface. One can construct models that distinguish between positively and negatively charged NPs, and the same exponential decay $N_{k} \propto \mathrm{e}^{-B k} t$ and linear trend $M \propto t$ can be obtained. Here we just wanted to demonstrate that the exponential size distribution emerges from a simple picture relying on a possible role the charges play in the aggregation process.

On the basis of the above considerations, the adsorption mechanism can be illustrated as in Figure 5.

The size distribution and the relative size could affect film formation. Previous studies have shown in a liquid phase that if the size distributions of the oppositely charged nanoparticles fully overlap, the nanoparticles can self-assemble into stable nanocrystals. However, if the average sizes of oppositely charged particles are different, this can provide formation of not-well-defined crystals. ${ }^{30}$ Changes in relative size can also affect the mechanism through the interplay between the electrostatic and van der Waals interactions, ${ }^{31}$ and this will be the topic of further investigations.

\section{CONCLUSIONS}

We investigated alternating adsorption of oppositely charged gold NPs (stabilized with self-assembled monolayers of positively charged TMA and negatively charged MUA) onto a negatively charged substrate. We used optical waveguide ligthmode spectroscopy (OWLS) to study the kinetic process of deposition of NPs onto the substrate surface. This method provides a convenient means for such investigations owing to a label-free surface. Our results revealed that already adsorbed positively charged particles considerably increase the adsorption of negatively charged particles. Investigation of the morphology of the created surface by AFM revealed that deposited NPs form clusters with various sizes and heights.

Kinetic information on cluster formation from nanoobjects can help to design and engineer nanostructured films and materials by self-assembly. Our results prove that label-free optical biosensors, so far explored to monitor proteins and living cells, ${ }^{21,27,28,32-34}$ could also be useful in investigating selfassembly processes in nanotechnology, especially when the kinetics of adsorption of nanoscopic components play a crucial role in the formation of nanostructured materials.

\section{AUTHOR INFORMATION}

\section{Corresponding Authors}

*E-mail grzybor@northwestern.edu.

*E-mail horvathr@mfa.kfki.hu.

\section{Notes}

The authors declare no competing financial interest.

\section{ACKNOWLEDGMENTS}

This work was supported by the Hungarian Scientific Research Fund (OTKA K104666) and by the Lendület (Momentum) program of the Hungarian Academy of Sciences and was realized in the frame of TÁMOP 4.2.4.A/2-11-1-2012-0001, National Excellence Program: Elaborating and operating an inland student and researcher personal support system convergence program. The project was subsidized by the European Union and cofinanced by the European Social Fund. The synthesis of thiols and the nanosynthesis for all nanoparticles used in this work was supported by the NonEquilibrium Energy Research Center, which is one of the Energy Frontier Research Centers established and funded by the U.S. Department of Energy.

\section{REFERENCES}

(1) Talapin, D. V.; Lee, J. S.; Kovalenko, M. V.; Shevchenko, E. V. Prospects of colloidal nanocrystals for electronic and optoelectronic applications. Chem. Rev. 2010, 110, 389-458.

(2) Lee, D.; Rubner, M. F.; Cohen, R. E. All-nanoparticle thin-film coatings. Nano Lett. 2006, 6, 2305-2312.

(3) Brust, M.; Bethell, D.; Kiely, C. J.; Schiffrin, D. J. Self-assembled gold nanoparticle thin films with nonmetallic optical and electronic properties. Langmuir 1998, 14, 5425-5429.

(4) Bigioni, T. P.; Lin, X. M.; Nguyen, T. T.; Corwin, E. I.; Witten, T. A.; Jaeger, H. M. Kinetically driven self-assembly of highly ordered nanoparticle monolayers. Nat. Mater. 2006, 5, 265-270.

(5) Krasteva, N.; Besnard, I.; Guse, B.; Bauer, R. E.; Mullen, K.; Yasuda, A.; Vossmeyer, T. Self-assembled gold nanoparticle/ dendrimer composite films for vapor sensing applications. Nano Lett. 2002, 2, 551-555.

(6) Smoukov, S. K.; Bishop, K. J. M.; Kowalczyk, B.; Kalsin, A. M.; Grzybowski, B. A. Electrostatically "patchy" coatings via cooperative adsorption of charged nanoparticles. J. Am. Chem. Soc. 2007, 129, $15623-15630$.

(7) Tretiakov, K. V.; Bishop, K. J. M.; Kowalczyk, B.; Jaiswal, A.; Poggi, M. A.; Grzybowski, B. A. Mechanism of the cooperative adsorption of oppositely charged nanoparticles. J. Phys. Chem. A 2009, 113, 3799-3803.

(8) Wang, Y.; Angelatos, A.S.; Caruso, F. Template synthesis of nanostructured materials via layer-by-layer assembly. Chem. Mater. 2008, 20, 848-858.

(9) Jiang, C. Y.; Tsukruk, V. V. Freestanding nanostructures via layerby-layer assembly. Adv. Mater. 2006, 18, 829-840.

(10) Jaworek, A. Micro- and nanoparticle production by electrospraying. Powder Technol. 2007, 176, 18-35.

(11) Decher, G.; Hong, J. D. Buildup of ultrathin multilayer films by a self-assembly process: II. Consecutive adsorption of anionic and cationic bipolar amphiphiles and polyelectrolytes on charged surfaces. Ber. Bunsen-Ges. 1991, 95, 1430-1434.

(12) Lavalle, Ph.; Gergely, C.; Cuisinier, F. J. G.; Decher, G.; Schaaf, P.; Voegel, J. C.; Picart, C. Comparison of the structure of polyelectrolyte multilayer films exhibiting a linear and an exponential growth regime: An in situ atomic force microscopy study. Macromolecules 2002, 35, 4458-4465.

(13) Ladam, G.; Schaad, P.; Voegel, J. C.; Schaaf, P.; Decher, G.; Cuisinier, F. In situ determination of the structural properties of 
initially deposited polyelectrolyte multilayers. Langmuir 2000, 16, 1249-1255.

(14) Tettey, K. E.; Ho, J. W. C.; Lee, D. Modulating layer-by-layer assembly of oppositely charged nanoparticles using a short amphiphilic molecule. J. Phys. Chem. C 2011, 115, 6297-6304.

(15) Srivastava, S.; Kotov, NA. Composite layer-by-layer (LbL) assembly with inorganic nanoparticles and nanowires. Acc. Chem. Res. 2008, 41, 1831-1841.

(16) Poon, Z.; Chang, D.; Zhao, X.; Hammond, P. T. Layer-by-layer nanoparticles with a $\mathrm{pH}$-sheddable layer for in vivo targeting of tumor hypoxia. ACS Nano 2011, 5, 4284-4292.

(17) Pál, E.; Sebok, D.; Hornok, V.; Dékány, I. Structural, optical, and adsorption properties of $\mathrm{ZnO}(2) /$ poly (acrylic acid) hybrid thin porous films prepared by ionic strength controlled layer-by-layer method. $J$. Colloid Interface Sci. 2009, 332, 173-82.

(18) Kalsin, A. M.; B. Kowalczyk, B.; Smoukov, S. K.; Klajn, R.; Grzybowski, B. A. Ionic-like behavior of oppositely charged nanoparticles. J. Am. Chem. Soc. 2006, 128, 15046-15047.

(19) Kalsin, A. M.; Kowalczyk, B.; Wesson, P.; Paszewski, M.; Grzybowski, B. A. Studying the thermodynamics of surface reactions on nanoparticles by electrostatic titrations. J. Am. Chem. Soc. 2007, 129, 6664-6665.

(20) Orgovan, N.; Patko, D.; Hos, C.; Kurunczi, S.; Szabó, B.; Ramsden, J. J.; Horvath, R. Sample handling in surface sensitive chemical and biological sensing: A practical review of basic fluidics and analyte transport. Adv. Colloid Interface Sci. 2014, 211, 1-16.

(21) Vörös, J.; Ramsden, J. J.; Csúcs, G.; Szendrő, I.; De Paul, S. M.; Textor, M.; Spencer, N. D. Optical grating coupler biosensors. Biomaterials 2010, 23, 3699-3710.

(22) Constable, E. C.; Harverson, P.; Ramsden, J. J. Adsorption of ruthenadendrimers to silica-titania surfaces studied by optical waveguide lightmode spectroscopy (OWLS). Chem. Commun. 1997, 16831684.

(23) Wang, Y.; Huang, C. J.; Jonas, U.; Wei, T.; Dostalek, J.; Knoll, W. Biosensor based on hydrogel optical waveguide spectroscopy. Biosens. Bioelectron. 2010, 25, 1663-1668.

(24) Ramsden, J. J. Optical biosensors. J. Mol. Recognit. 1997, 10, $109-120$.

(25) Ramsden, J. J.; Máté, M. Kinetics of monolayer particle deposition. J. Chem. Soc., Faraday Trans. 1998, 94, 783-788.

(26) Picart, C.; Ladam, G.; Senger, B.; Voegel, J.-C.; Schaaf, P.; Cuisinier, F. J. G.; Gergely, C. Determination of structural parameters characterizing thin films by optical methods: A comparison between scanning angle reflectometry and optical waveguide lightmode spectroscopy. J. Chem. Phys. 2001, 115, 1086-1094.

(27) Höök, F.; Vörös, J.; Rodahl, M.; Kurrat, R.; Böni, P.; Ramsden, J. J.; Textor, M.; Spencer, N. D.; Tengvall, P.; Gold, J.; Kasemo, B. A comparative study of protein adsorption on titanium oxide surfaces using in situ ellipsometry, optical waveguide lightmode spectroscopy, and quartz crystal microbalance/dissipation. Colloids Surf., B 2002, 24, $155-170$.

(28) Máté, M.; Ramsden, J. J. Addition of particles of alternating charge. J. Chem. Soc., Faraday Trans. 1998, 94, 2813-2816.

(29) Gardiner, C. W. Handbook of Stochastic Methods for Physics, Chemistry and the Natural Sciences; Springer-Verlag: Berlin, 1985.

(30) Kowalczyk, B.; Kalsin, A. M.; Orlik, R.; Bishop, K. J. M.; Patashinski, A. Z.; Mitus, A.; Grzybowski, B. A. Size-selection during crystallization of oppositely charged nanoparticles. Chem.-Eur. J. 2009, 15, 2032-2035.

(31) Zhang, R.; Jha, P. K.; Olvera de la Cruz, M. Non-equilibrium ionic assemblies of oppositely charged nanoparticles. Soft Matter 2013, 9, 5042-5051.

(32) Dee Feijter, J. A.; Benjamins, J.; Veer, F. A. Ellipsometry as a tool to study the adsorption behavior of synthetic and biopolymers at the air-water interface. Biopolymers 1978, 17, 1759-1772.

(33) Kovacs, N.; Patko, D.; Orgovan, N.; Kurunczi, S.; Ramsden, J. J.; Vonderviszt, F.; Horvath, R. Optical anisotropy of flagellin layers: In situ and label-free measurement of adsorbed protein orientation using OWLS. Anal. Chem. 2013, 85, 5382-5389.
(34) Orgovan, N.; Salánki, R.; Sándor, N.; Bajtay, Z.; Erdei, A.; Szabó, B.; Horvath, R. In-situ and label-free optical monitoring of the adhesion and spreading of primary monocytes isolated from human blood: Dependence on serum concentration levels. Biosens. Bioelectron. 2013, 54, 339-344. 